\title{
Lifestyle and precision diabetes medicine: will genomics help optimise the prediction, prevention and treatment of type 2 diabetes through lifestyle therapy?
}

\author{
Paul W Franks ${ }^{1,2,3} \cdot$ Alaitz Poveda $^{1}$
}

Received: 5 October 2016 / Accepted: 16 December 2016 / Published online: 25 January 2017

(C) The Author(s) 2017. This article is published with open access at Springerlink.com

\begin{abstract}
Precision diabetes medicine, the optimisation of therapy using patient-level biomarker data, has stimulated enormous interest throughout society as it provides hope of more effective, less costly and safer ways of preventing, treating, and perhaps even curing the disease. While precision diabetes medicine is often framed in the context of pharmacotherapy, using biomarkers to personalise lifestyle recommendations, intended to lower type 2 diabetes risk or to slow progression, is also conceivable. There are at least four ways in which this might work: (1) by helping to predict a person's susceptibility to adverse lifestyle exposures; (2) by facilitating the stratification of type 2 diabetes into subclasses, some of which may be prevented or treated optimally with specific lifestyle interventions; (3) by aiding the discovery of prognostic biomarkers that help guide timing and intensity of lifestyle interventions; (4) by predicting treatment response. In this review we overview the rationale for precision diabetes medicine, specifically as it relates to lifestyle; we also scrutinise existing evidence, discuss the barriers germane to research in this field and consider how this work is likely to proceed.
\end{abstract}

Electronic supplementary material The online version of this article (doi:10.1007/s00125-017-4207-5) contains a slideset of the figures for download, which is available to authorised users.

Paul W Franks

paul.franks@med.lu.se

1 Department of Clinical Sciences, Genetic and Molecular Epidemiology Unit, Clinical Research Centre, Lund University, Jan Waldenströms gata 35, Skåne University Hospital, Malmö SE-20502, Sweden

2 Department of Public Health and Clinical Medicine, Section for Medicine, Umeå University, Umeå, Sweden

3 Department of Nutrition, Harvard T.H. Chan School of Public Health, Boston, MA, USA
Keywords Biomarkers $\cdot$ Lifestyle $\cdot$ Precision medicine $\cdot$ Review $\cdot$ Type 2 diabetes
Abbreviations
DPP Diabetes Prevention Program
MZ Monozygotic
NIH National Institutes of Health

\section{Introduction}

The major developments in genomic technologies and their application to large, well characterised collections of samples have led to the generation of extensive new knowledge about disease biology. This has inspired new avenues for type 2 diabetes prevention, treatment and cure that are inherent to the concept of precision medicine. According to the National Research Council [1], precision medicine is not intended to involve the complete personalisation of medical devices and therapies; instead, it should focus on the classification of 'individuals into subpopulations that differ in their susceptibility to a particular disease, in the biology and/or prognosis of those diseases they may develop, or in their response to a specific treatment' with the expectation that 'preventive or therapeutic interventions can then be concentrated on those who will benefit, sparing expense and side effects for those who will not'. By this and most other definitions, precision medicine focuses on applying biomarker technologies to the individual patient to help improve prediction and assessment of: (1) risk-factor susceptibility; (2) disease stratification; (3) prognosis; and (4) treatment response.

Our understanding of type 2 diabetes pathobiology has improved dramatically recently, owing largely to a quantum leap in human genome sequencing, precipitated by ground-breaking achievements made in the preceding century, including 
mapping of the Drosophila genome [2] and the structural characterisation of DNA [3]. These were the foundations for human genome sequencing $[4,5]$ and affordable technologies for highresolution characterisation of the genome, metagenome, epigenome, transcriptome, proteome, and metabolome. Combined with novel bioinformatics and the emergence of large global collaborative networks, exciting possibilities have emerged for the prediction and prevention of disease in ways that are more personal and precise than ever before.

Genetic variation is quite literally the starting point of the biological cascade that underpins phenotypic expression (known as the 'central dogma of molecular biology') [6]. But for complex diseases like type 2 diabetes, genetics is by no means the absolute determinant, and thus an enormous amount of downstream work remains before we will adequately understand how genetic and lifestyle factors (e.g. nutrition, exercise, medications and stress) work jointly to affect gene transcription and translation, and phenotypic expression (Fig. 1). The model is thus one of primers and catalysts: environmental triggers in the context of genetic predisposition.

Given that significant changes in human genetic variation manifest over many generations, the consensus is that the global surge in type 2 diabetes prevalence is caused predominantly by the rapid and widespread adoption of obesogenic lifestyles. Metabolic dyshomeostasis is a common consequence of unhealthy lifestyles, driven by disturbed substrate production and/or metabolism in the liver, skeletal muscle and adipose tissue, or by interfering with the synthesis, secretion or action of insulin. However, diabetes pathophysiology is complex and heterogeneous with multiple feedback loops, such that people vary in susceptibility to risk factors and response to therapies, and the molecular defects that cause the disease in a given patient are rarely known. Nevertheless, the measurement or prediction of primordial factors might

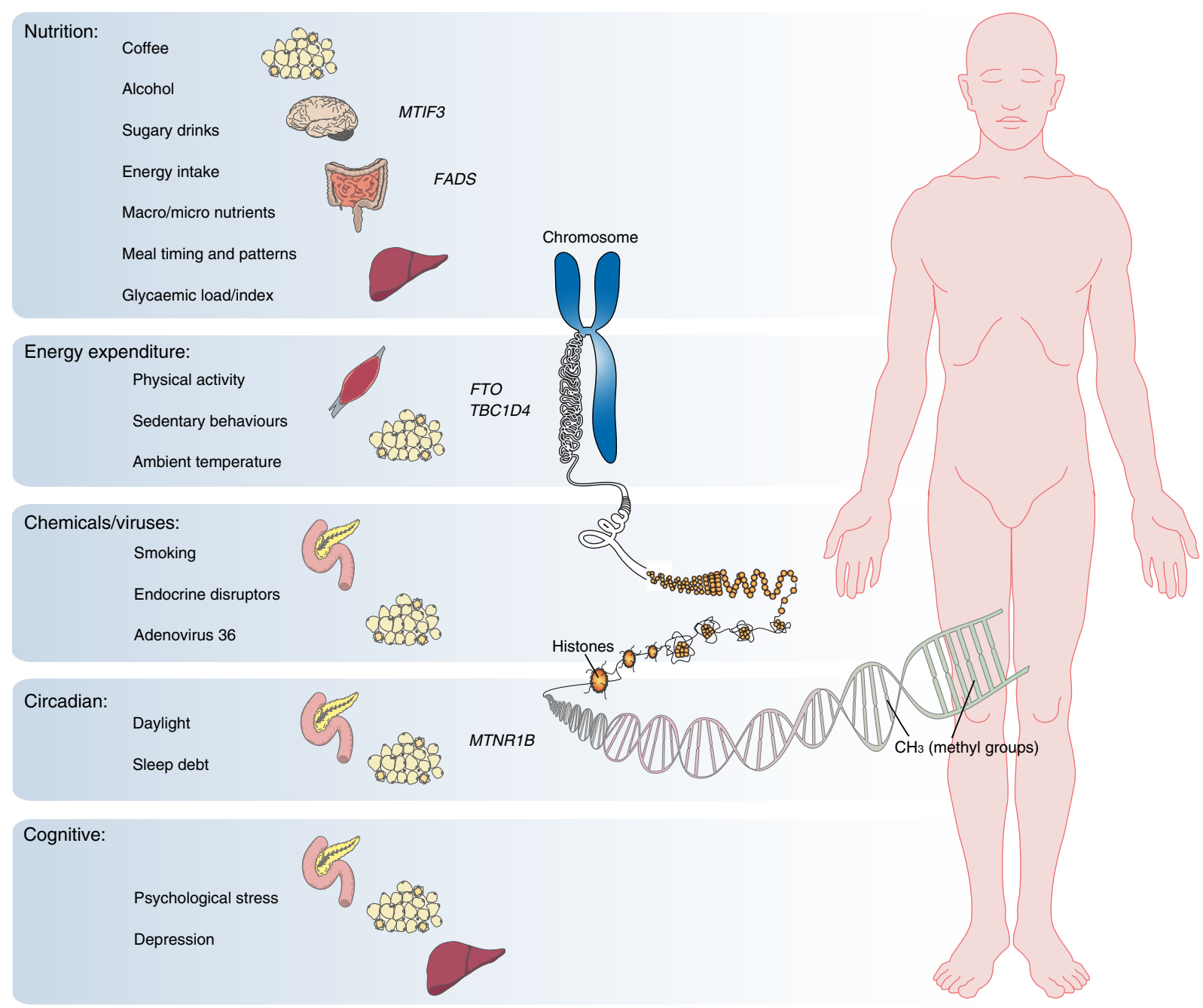

Fig. 1 Type 2 diabetes results from the complex interplay between environmental and genomic factors. The model is thus one of primers and catalysts, whereby environmental triggers act against a backdrop of genetic susceptibility to affect the transcriptional and regulatory processes that cause diabetes (e.g. through methylation, chromatin remodelling or histone modifications). The figure shows the key lifestyle risk factors, candidate loci (with evidence of gene-lifestyle interactions) and target organs purported to affect adiposity and/or glycaemic control 
facilitate more effective diabetes prevention if they helped determine the specific risk factors to which a person is susceptible and the therapies they are likely to respond well to.

\section{Lifestyle in type 2 diabetes}

In most people at risk of or with type 2 diabetes, prognosis can be improved by enhancing peripheral insulin sensitivity. Although oral glucose-lowering agents are often used for this purpose, reducing energy intake and increasing non-resting energy expenditure (physical activity) are highly effective first line therapeutic options, with associated reduced lipid content in or around adipose, liver and muscle tissue being pivotal to this process.

Although reduction of energy intake and an increase in physical activity can both cause weight loss, they do so through contrasting states of low and high metabolic turnover respectively, which involve the activation of different molecular pathways and processes that encompass both common and unique corresponding health benefits. Accordingly, some patients will respond well and others poorly to the same intervention, with response being determined in part by individual biology, but also by psychosocial factors that influence adherence and perceived success.

Non-resting energy expenditure Non-resting energy expenditure constitutes activities that are either structured, with the intent of improving fitness or sports skills (i.e. exercise training), or activities that are not explicitly intended as exercise (e.g. active commuting, gardening, dog walking), as well as subconscious movements (e.g. fidgeting). Regardless of mode, regular physical activity can improve glucose homeostasis through insulin-dependent mechanisms (e.g. by improving the sensitivity of peripheral tissue to insulin) and insulin-independent mechanisms (e.g. muscle contraction, shear stress, reductions in hepatic glucose production), thereby reducing pancreatic beta cell stress, and helping to prevent or slow progression of diabetes.

Diet Diet also plays many complex roles in metabolic homeostasis. Frequently, the perceived link between diet and type 2 diabetes is through excess energy intake that leads to obesity, and over-consumption of refined carbohydrates that rapidly raises blood glucose and places a compensatory demand on the beta cells for endogenous insulin. However, there are many other roles diet plays in affecting diabetes risk: zinc, for example, regulates insulin storage in the secretory granules of the pancreatic beta cells, and functional variants within $S L C 30 A 8$, encoding a zinc transporter, affect this process [7]; and long chain polyunsaturated fatty acids are ligands for fatty acid receptors, like peroxisome proliferator-activated receptor gamma (PPAR $\gamma)$ [8]. Overall diet quality (e.g. Mediterranean diet) is also an important feature of many successful diabetes prevention programmes [9].

Body composition Adequately characterising lifestyle exposures is thus important, but so too is how body corpulence is defined. Emphasis is often on total adiposity (e.g. weight change); however, the regional distribution of adipose tissue (particularly when it is deep within the abdominal cavity and within or around the liver, heart and pancreas) [10] and the patterns of change in response to an intervention [11], are likely to elucidate diabetes aetiology better than weight change per se. The emergence of optical triangulation 3D scanning technologies that allow frequent assessments of body form to be captured quickly, safely and at relatively low cost, may facilitate this process [12].

Implementing structured lifestyle interventions for diabetes prevention in clinical practice is cost effective [13]. Behavioural assessment is a critical part of this process, helping to identify key risk factors, determine appropriate intervention targets and gauge adherence. Ideally such assessments would be performed through the diabetes care system, but time and cost limitations are major barriers to the implementation of lifestyle medicine and careful lifestyle assessments are rarely undertaken in practice [14]. This is unfortunate, as appropriate lifestyle monitoring and tailoring of lifestyle advice may help further improve the efficacy and cost effectiveness of lifestyle medicine.

\section{How precision lifestyle medicine might work}

Precision medicine in type 2 diabetes is very much at a theoretical stage, particularly as it relates to personalised lifestyle therapy. However, its successes in other diseases and with pharmacotherapy offer a glimpse of how tailored lifestyle advice for type 2 diabetes prevention, guided by personal genomic data, might be achievable (Fig. 2 and text box: Precision lifestyle medicine in type 2 diabetes):

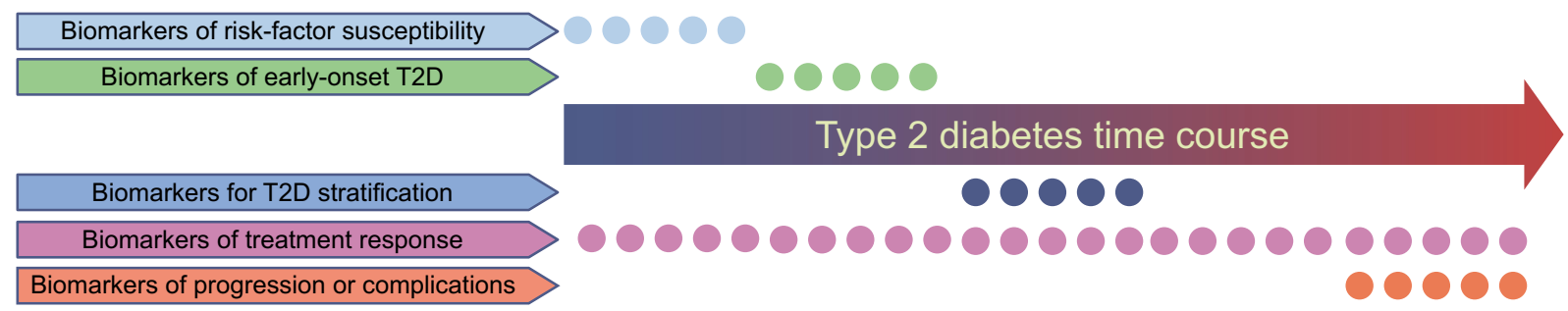

Fig. 2 Precision medicine for type 2 diabetes. A schematic showing key time points for intervention in the course of type 2 diabetes (T2D) pathophysiology where precision lifestyle medicine might play a role 
Precision lifestyle medicine in type 2 diabetes

Why?

Diabetes pathophysiology is complex and heterogeneous; people vary in susceptibility to risk factors and response to therapies

Reducing energy intake and increasing physical activity are highly effective therapeutic options to improve glucose homeostasis

Variability in the response to the same lifestyle intervention is partly determined by individual biology and psychosocial factors that influence adherence and perceived success

Tailoring of lifestyle advice may improve efficacy and cost effectiveness of lifestyle medicine

How?

Biomarkers for stratification Type 2 diabetes is complex and the diagnosis does not take into account pathophysiology. There is no clinically accessible causal biomarker for type 2 diabetes. Subclassification may guide therapeutic decisions; different lifestyle interventions would be more or less effective at preventing or treating type 2 diabetes subtype

Biomarkers for therapies that target specific genetic defects In type 2 diabetes, a rare example of variation at a specific gene that has been treated with a natural chemical compound (yohimbine) concerns $A D R A 2 A$. The design of drugs that target specific pathogenic mutations may improve the success of precision diabetes medicine

Biomarkers of early onset, progression or complications These biomarkers would be highly relevant in diabetes medicine, as carriers of these biomarkers might be prioritised for intensive lifestyle therapy

Biomarkers of risk factor susceptibility and treatment response Type 2 diabetes guidelines concerning diet and exercise are relatively generic and there is considerable variation in how people respond to lifestyle therapies. Predicting a patient's response to treatment could help optimise the management of their disease

Biomarkers for type 2 diabetes stratification An excellent example of precision diabetes medicine can be found in MODY. The disease, often misdiagnosed as type 1 diabetes owing to the young age of onset and insulin deficiency, can be accurately and precisely diagnosed using genetic screening, leading to subclassification and highly effective targeted drug therapy.

From an aetiological perspective, type 2 diabetes is far more complex than MODY, and in type 2 diabetes the direct diagnostic assay is restricted to blood glucose (or glycosylated haemoglobin). However, elevations in blood glucose are usually the consequence of other cellular defects in the liver, pancreas, skeletal muscle and other peripheral tissues, primarily affecting the endogenous production of glucose and insulin and the rate of glucose metabolism. There is no clinically accessible causal biomarker for type 2 diabetes, although markers of beta cell function are often used to help diagnose other causes of abnormal blood glucose concentrations. Thus, diagnosing type 2 diabetes and distinguishing it from other causes of elevated blood glucose is challenging and rarely elucidates the primary cause of the disease.

Subclassification of type 2 diabetes using biomarkers and other information might not pinpoint specific molecular causes, but it may bring the diagnosis closer to that point and guide therapeutic decisions. In a recent study conducted in the USA, the use of electronic medical records for the classification of type 2 diabetes into subtypes (coinciding with cardiovascular diseases, neurological diseases, allergies and HIV infections) that were subsequently genetically characterised provides an example of how type 2 diabetes diagnoses might be refined through genotype-guided patient stratification [15]. Moreover, amongst the millions of people diagnosed with type 2 diabetes annually undoubtedly reside those with rare monogenetic disorders that appear like type 2 diabetes, yet are caused by rare single gene mutations. These mutations might be targetable with specific treatments, such as recently described for the $S U R 1$ (also known as $A B C C 8$ ) locus [16]. Conceivably, different lifestyle interventions, 
particularly those with a nutritional emphasis, would be more or less effective at preventing or treating each type 2 diabetes subtype, thus providing avenues for personalised lifestyle therapy.

Biomarkers for therapies that target specific genetic defects Some of the most remarkable successes in precision medicine to date have involved the design of drugs that target specific pathogenic mutations. Two striking examples are drugs for treating chronic myelogenous leukaemia and lung adenocarcinoma, imatinib (Glivec/Gleevec) and crizotinib (Xalkori), respectively. Imatinib targets the protein product of a novel fusion gene, $B C R-A B L$ [17], whereas crizotinib targets a genetic abnormality (a fusion gene called EML4$A L K)$ caused by the inversion of the anaplastic lymphoma kinase $(A L K)$ gene [18]. In type 2 diabetes, a rare example of a specific gene defect that has been successfully treated with naturally occurring chemical compounds is that of the $\alpha 2 \mathrm{~A}$ adrenergic-receptor $\left(\alpha_{2 \mathrm{~A}} \mathrm{AR}\right)$ encoding gene, $A D R A 2 \mathrm{~A}$ [19]. Here, an $A D R A 2 A$ variant causes type 2 diabetes as a result of impaired insulin secretion owing to receptor overexpression; treatment with the naturally occurring indole alkaloid, yohimbine (a chemical compound extracted from Pausinystalia johimbe tree bark) blocks the receptor and improves insulin secretion.

Biomarkers of early-onset, progression or complications Biomarkers of the early-onset or progression of type 2 diabetes, or of the onset of diabetes-associated complications, would be highly relevant for diabetes therapy. Carriers of these biomarkers might be prioritised for intensive lifestyle therapy before or soon after the disease is manifest, much as people with a family history of diabetes are treated today. However, no tangible examples of these biomarkers currently exist.

\section{Biomarkers of risk-factor susceptibility and treatment re-} sponse Type 2 diabetes guidelines concerning diet and exercise are relatively generic. Such guidelines are typically derived from epidemiological studies on the risk attributable to modifiable lifestyle exposures and clinical trials showing that intervening with those same factors reduces risk. Importantly, these data reflect population averages, often with wide confidence intervals, indicating that, although targeting risk factors diminishes type 2 diabetes risk on average, there are people within intervention groups who improve greatly (so called 'super-responders'), and others who may not improve at all (so called 'non-responders') or whose condition worsens (so called 'adverse responders'). One of the first studies on blood glucose variability in response to chronic exercise showed that although mean insulin sensitivity increased by
$10 \%$ following the intervention $42 \%$ of the participants experienced no change or became more insulin resistant [20]. According to a recent review, the proportion of nonresponders to exercise training regarding glucose homeostasis ranged between $7 \%$ and $63 \%$ [21] and the number of adverse responders averaged $8.4 \%$ [22]. The relatively high proportion of people who do not appear to respond well to exercise has motivated the search for the underlying mechanisms [23], with the assumption that genetic and epigenomic variation play a key role. However, as we discuss later, how much of this variability is of biological origin and how much is driven by other factors remains unclear.

\section{Evidence base: strengths and weakness}

Early twin and family studies showed that response to diet and exercise interventions vary to a significantly greater extent between sibships and pedigrees than within them, suggesting a heritable component to some treatment response phenotypes [24-26]. A 100-day overfeeding protocol conducted in 12 pairs of monozygotic (MZ) male twins showed, for example, that the gain in fat mass was roughly three times more similar within the MZ twin pairs than between non-twins [26]. Elsewhere, a study conducted to investigate the response to a negative energy balance in seven pairs of MZ male twins found that the variance in change in fat mass was 14.1 times more similar within than between twin pairs [25].

When used to model interactions between common genetic variants and lifestyle exposures, data from observational studies can generate hypotheses relevant to treatment response and risk-factor susceptibility. However, the inherent limitations of epidemiology (chance, bias, confounding), the difficulties in accurately and precisely assessing phenotypes and lifestyle behaviours in freeliving populations, as well as challenges that specifically hinder the estimation of interaction effects in observational data (e.g. heteroscedasticity and scale dependency) necessitate caution when interpreting the causal relevance of observational data. In interaction studies, the imperative of replication has been especially difficult to achieve owing to winner's curse, heterogeneous study designs, environmental idiosyncrasies, etc. (see [27]). Nevertheless, a complete absence of replication in the face of concerted, adequately powered attempts undermines the value of the original findings, as they likely reflect idiosyncratic effects or false-positives.

There is little extensively replicated epidemiological evidence of gene-lifestyle interactions, with the exception of those at the FTO locus (see text box: What we know about the FTO-physical activity interaction). Soon 
after the finding that FTO variation affects obesity risk, data emerged from two epidemiological studies showing that FTO variation may modify the relationship between physical activity and estimates of adiposity in European [28, 29] and North American [30] adults. An analysis of clinical trial data from the Diabetes Prevention Program (DPP) found no evidence that FTO modified the effects of lifestyle intervention on weight loss, although there was weak evidence that an interaction might affect subcutaneous adipose mass [31]. Many studies followed, but with mixed results; therefore, we undertook a large meta-analysis $(N=220,000)$ to test the hypothesis, which confirmed the presence of an interaction [32]. Those findings were recently extended in the UK Biobank [33], where statistically robust interaction effects were reported between the FTO variant and physical activity, frequency of alcohol consumption, sleep duration, diet and salt consumption. Cross-sectional analyses focusing on lifestyle interactions with genetic risk scores comprised of other obesity-associated loci have also been widely replicated, although the extent to which those interactions are driven by FTO is rarely described.

\section{What we know about the FTO-physical activity interaction}

Observational studies can generate hypotheses for treatment response and risk-factor susceptibility. However, replicated epidemiological evidence of gene-lifestyle interactions is scarce. An exception is the interaction of FTO and physical activity in obesity risk

Although the epidemiological evidence of this interaction is strong [32, 33], a meta-analysis of clinical trial data [40] found no evidence of interaction between FTO and lifestyle in weight change

The opposing conclusions may be explained by differences in: (1) study setting: the observational analyses are cross-sectional while the clinical trials are prospective; (2) study duration: the observational analyses reflect long term exposures, whereas the exposures in clinical trials are shorter duration; (3) study size: the epidemiological studies are large $(N=100,000$ 200,000 ), whilst the clinical trials are smaller $(N=9563)$ and possibly underpowered to detect interaction effects; (4) sample type: the clinical trials were conducted in more highly selected populations than the observational studies
While the epidemiological data seem compelling, it is predominantly cross-sectional and evidence of gene-lifestyle interactions in large observational datasets may be biased or confounded. Hence, epidemiological evidence of gene-lifestyle interactions should not only be replicated but also supported by other types of causal evidence before exploiting the findings for the purposes of genotype-guided lifestyle prescription. There are some functional data positioning FTO as a plausible candidate for lifestyle interactions (e.g. FTO encodes a 2-oxoglutarate oxygenase that is highly expressed in hypothalamic nuclei in mice and humans [34], and phosphocreatine and inorganic phosphate levels have been shown to recover more rapidly following exhaustive exercise in FTO (rs9939609) risk allele carriers [35]), but the discovery of long-range activation of IRX3 and IRXS by FTO [36, 37] suggests that simple interpretations about how FTO and lifestyle interact are unlikely to be accurate.

Nevertheless, while understanding the functional basis of interactions is desirable, the absence of this knowledge does not preclude using evidence of gene-lifestyle interactions for precision medicine, whereas demonstrating cause and effect in trials is essential. A recent systematic review of selected published trial data concluded that $F T O$ variants modify weight loss in response to lifestyle interventions [38]. However, the meta-analysis of published data for gene-lifestyle interactions may be biased [39], and a subsequent meta-analysis of clinical trial data, which adopted a more rigorous and more inclusive approach involving de novo, standardised interaction analyses, found no evidence of interaction between FTO and lifestyle [40]. A recent clinical trial analysis (DPP and Look AHEAD [The Action for Health in Diabetes] studies) that focused on the spectrum of BMIassociated variants reached similar conclusions for FTO and almost all other variants assessed; the exception was for MTIF3, which showed evidence of gene-lifestyle interactions in the trials [41], as well as in epidemiological cohorts [42].

Although the starkly opposing conclusions drawn about FTO from the observational and clinical trial data appear contradictory, they are potentially reconcilable. For example, the observational analyses [32] are cross-sectional and may reflect the modifying effects of very long-term lifestyle exposures and outcomes, whereas the clinical trials [40] are prospective and confined to a relatively short intervention period (ranging from 8 weeks to 3 years). Moreover, the epidemiological studies are large $(N=100,000-200,000)$ and apparently adequately powered, whereas the trials analysis is an order of magnitude smaller $(N=9563)$, may have been underpowered to detect interaction effects, and was conducted in very selected populations compared with the observational studies (Fig. 3). Nevertheless, the absence of an interaction effect in the trials indicates that, for the benefit of enhancing weight loss for diabetes prevention, there may be little clinical value in tailoring common lifestyle interventions to FTO genotype. 


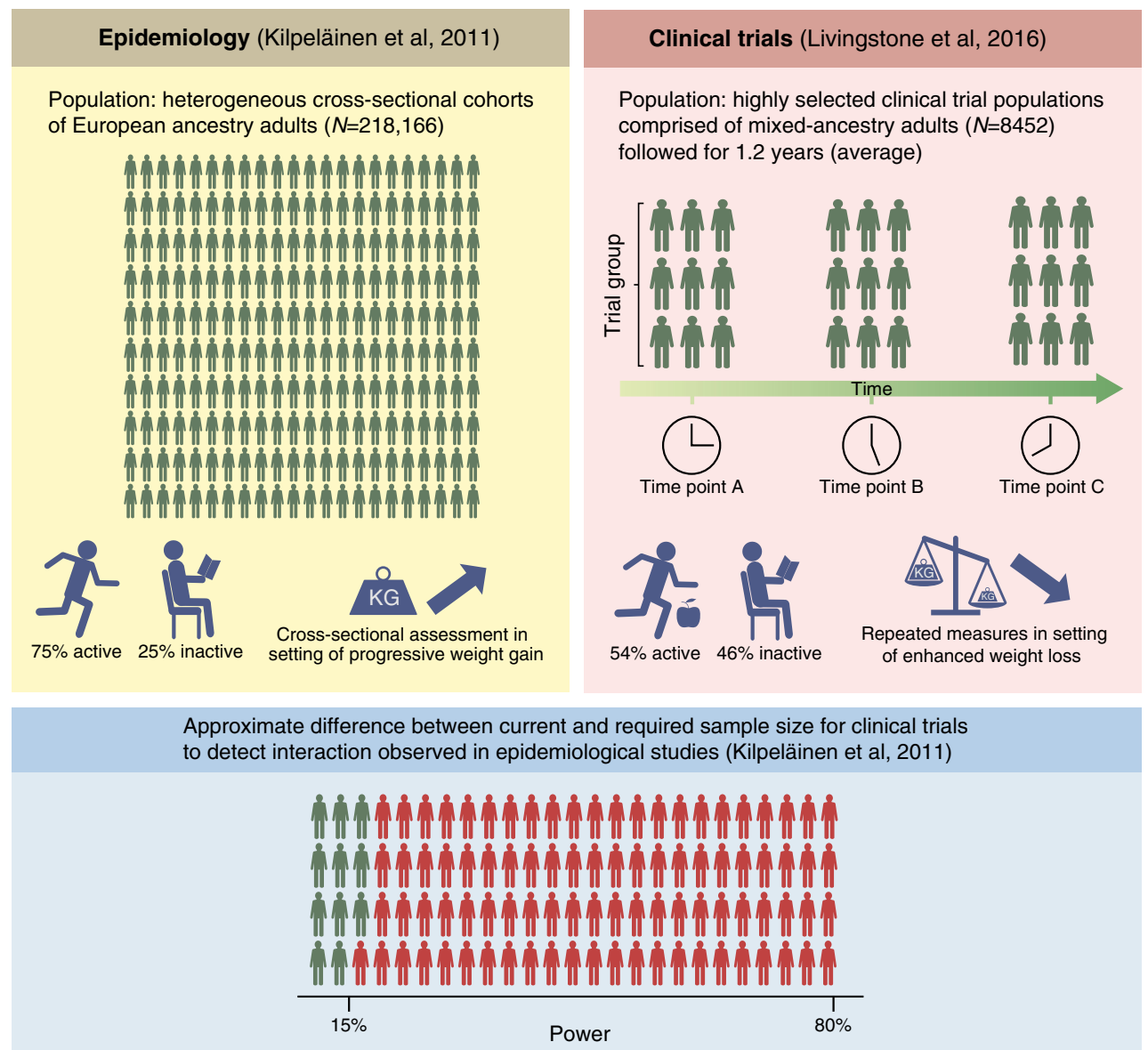

Fig. 3 Estimating the required power for clinical trials focused on interaction effects of diabetes risk factors that have been previously reported in epidemiological studies. The figure compares two core studies focused on the interaction of FTO variants and lifestyle in obesity. We sought to estimate the power that the sample size reported by Livingstone et al [40] had to detect the interaction of the FTO variant and physical activity in obesity, as previously described in Kilpeläinen et al [32]. The conclusions regarding power and sample size in trials in this figure are

\section{The future}

Determining the causal effects of lifestyle, even in randomised controlled trials, is much more challenging than for drug therapies, not least because most lifestyle interventions cannot be masked and no placebo exists. Thus, a participant's behaviours during a lifestyle trial that affect the trial's outcomes may change as a consequence of the intervention in ways that are not expected or measured. This phenomenon was first described in a study of older men and women (58-78 years old) who underwent an 8 week supervised aerobic training programme (cycling three times per week) [43]. Objective assessments of total energy expenditure were made using doubly labelled water, resting metabolic rate by respiratory gas exchange, and physical activity energy expenditure using the latter values combined with information about sleep and exercise. The authors predicated on the assumption that the interaction effect reported in the cross-sectional epidemiological analysis [32] can be applied to the setting of a randomised lifestyle intervention meta-analysis. However, there are several factors that are likely to confound this comparison; these are outlined in the figure. The given estimates of power and sample size are intended only to illustrate that the trials are likely to be substantially underpowered to observe previously reported interaction effects, rather than provide precise estimates of these variables

found that despite an increase in exercise energy expenditure of $628 \mathrm{~kJ} /$ day ( $150 \mathrm{kcal} /$ day), non-exercise-activity energy expenditure decreased proportionately and no overall change in total energy expenditure was hence observed.

When one considers that exercise interventions deployed in clinical trials typically occupy about 150 mins per week, or about $1.5 \%$ of the total time, it is possible that compensatory behaviours underlie much of the apparent heterogeneity in response. Even in tightly controlled inpatient studies, unintended variations in the participants' behaviours can hinder data interpretation. For example, in one of the studies cited above, 84 days of overfeeding with restricted physical activity resulted in an average weight gain of $8.1 \mathrm{~kg}$ (range: $4.3-13.3 \mathrm{~kg}$ ) [26]. The very lowest and highest weight gains suggest that the diet and exercise regime was not strictly followed, as a weight gain of $4.3 \mathrm{~kg}$ with this level of overfeeding accounts for only $40 \%$ of 
the predicted weight gain for that participant and would require energy expenditure equivalent to running $\sim 17-29 \mathrm{~km}$ each week during the intervention. By contrast, a weight gain of $13.3 \mathrm{~kg}$ would require complete indolence during the protocol. While individual biological variation in energy metabolism might explain some of these differences in weight gain, it seems probable that lack of adherence to the study protocol is the predominant explanation.

In current research and practice, lifestyle behaviours are frequently assessed through self-report methods, despite some key limitations [44]. However, numerous wearable technologies exist that are relatively inexpensive, suitable for long-term monitoring, and valid, albeit also with important caveats [45]. Nevertheless, modern wearables, used in combination with selfreport methods have tremendous potential to characterise healthrelated behaviours and exposures, as the continuous assessment of movement, sleep, temperature, blood glucose and other relevant factors is possible without apparently impacting behaviour [46]. The use of such devices in epidemiology and clinical trials is likely to be necessary to delineate biological drivers of risk-factor susceptibility and treatment response from other sources of error and bias that cause heterogeneity. The continuous assessment of quantitative phenotypes germane to a clinical trial's outcomes would also help overcome a further major limitation of some lifestyle trials that only assessed outcomes at enrolment and the trial's end. In those settings, regression dilution, which occurs when changes in quantitative traits are inferred from too few data points, generates error. Thus, continuous (or frequently repeated) assessments of the trial's quantitative outcomes would further help isolate response variability from error. In a recent eloquent study that focused on personalised diets, Zeevi and colleagues collected detailed diet data and objectively assessed physical activity and blood glucose in 800 Israeli adults during a 1 week observational phase. In combination with gut microbiota data, the authors were able to predict individual postprandial glucose excursions to specific foods and designed personalised diet interventions that improved glucose control [47]. This approach had the added benefit of allowing the background heterogeneity in the participants' lifestyles to be factored into the intervention design, further reducing error.

\section{Summary}

Although evidence of specific genetic loci that modulate risk-factor susceptibility and treatment response in type 2 diabetes is weak, the rationale, which is supported by data from twin and family studies, remains strong, motivating continued research in this area. For example, the National Institutes of Health's (NIH's) Common Fund initiative called the Molecular Transducers of Physical Activity (http://commonfund.nih. gov/MolecularTransducers), is a multimillion dollar funding programme focused on defining 'optimal physical activity recommendations for people at various stages of life' and developing 'precisely targeted regimens for individuals with particular health needs.' Nevertheless, this topic is clearly challenging, and to realise the vision of the NIH and others will likely require new study designs and analytical methods that overcome the major barriers to precision lifestyle medicine in type 2 diabetes.

Acknowledgements Some of the ideas outlined in this review were stimulated by discussions at the $91^{\text {st }}$ Berzelius Symposium on Precision Medicine in Type 2 Diabetes and Cardiovascular Disease, held in Båstad, Sweden (2016).

Funding PWF acknowledges funding for work related to this paper from the Swedish Heart-Lung Foundation, Novo Nordisk, the Swedish Research Council, the European Research Council (CoG2015681742 NASCENT) and the Innovative Medicines Initiative (DIRECT: grant agreement \#115317; RHAPSODY: grant agreement \#115881). AP was supported by a grant from the Swedish Research Council. The study sponsor was not involved in the design of the study; the collection, analysis, and interpretation of data; writing the report; or the decision to submit the report for publication.

Duality of interest PWF has been a paid member of advisory boards for Sanofi Aventis and Eli Lilly and has received research funding from Sanofi Aventis, Boehringer Ingelheim, Eli Lilly, Jansson, Servier and Novo Nordisk. AP declares that there is no duality of interest associated with her contribution to this manuscript.

Contribution statement PWF wrote the manuscript upon which AP provided critical input. Both authors read the manuscript and contributed to the final version. Both authors approved the version to be published.

Open Access This article is distributed under the terms of the Creative Commons Attribution 4.0 International License (http:// creativecommons.org/licenses/by/4.0/), which permits unrestricted use, distribution, and reproduction in any medium, provided you give appropriate credit to the original author(s) and the source, provide a link to the Creative Commons license, and indicate if changes were made.

\section{References}

1. National Research Council (US) Committee on A Framework for Developing a New Taxonomy of Disease (2011) Toward precision medicine: building a knowledge network for biomedical research and a new taxonomy of disease. National Academies Press, Washington, DC

2. Sturtevant AH (1917) Genetic factors affecting the strength of linkage in Drosophila. Proc Natl Acad Sci U S A 3:555-558

3. Watson JD, Crick FH (1953) Molecular structure of nucleic acids; a structure for deoxyribose nucleic acid. Nature 171:737-738

4. Venter JC, Adams MD, Myers EW et al (2001) The sequence of the human genome. Science 291:1304-1351

5. Lander ES, Linton LM, Birren B et al (2001) Initial sequencing and analysis of the human genome. Nature 409:860-921 
6. Crick F (1970) Central dogma of molecular biology. Nature 227: 561-563

7. Rutter GA, Chabosseau P, Bellomo EA et al (2016) Intracellular zinc in insulin secretion and action: a determinant of diabetes risk? Proc Nutr Soc 75:61-72

8. Schoonjans K, Staels B, Auwerx J (1996) Role of the peroxisome proliferator-activated receptor (PPAR) in mediating the effects of fibrates and fatty acids on gene expression. J Lipid Res 37:907-925

9. Salas-Salvado J, Bullo M, Babio N et al (2011) Reduction in the incidence of type 2 diabetes with the Mediterranean diet: results of the PREDIMED-Reus nutrition intervention randomized trial. Diabetes Care 34:14-19

10. Yaghootkar H, Lotta LA, Tyrrell J et al (2016) Genetic evidence for a link between favorable adiposity and lower risk of type 2 diabetes, hypertension, and heart disease. Diabetes 65:2448-2460

11. Delahanty LM, Pan Q, Jablonski KA et al (2014) Effects of weight loss, weight cycling, and weight loss maintenance on diabetes incidence and change in cardiometabolic traits in the diabetes prevention program. Diabetes Care 37:2738-2745

12. Loffler-Wirth H, Willscher E, Ahnert P et al (2016) Novel anthropometry based on 3D-Bodyscans applied to a large population based cohort. PLoS One 11, e0159887

13. Herman WH, Edelstein SL, Ratner RE et al (2013) Effectiveness and cost-effectiveness of diabetes prevention among adherent participants. Am J Manag Care 19:194-202

14. McTigue KM, Conroy MB, Bigi L, Murphy C, McNeil M (2009) Weight loss through living well: translating an effective lifestyle intervention into clinical practice. Diabetes Educ 35:199-208

15. Li L, Cheng WY, Glicksberg BS et al (2015) Identification of type 2 diabetes subgroups through topological analysis of patient similarity. Sci Transl Med 7:311ra174

16. Baier LJ, Muller YL, Remedi MS et al (2015) ABCC8 R1420H loss-of-function variant in a Southwest American Indian community: association with increased birth weight and doubled risk of type 2 diabetes. Diabetes 64:4322-4332

17. Druker BJ, Guilhot F, O'Brien SG et al (2006) Five-year follow-up of patients receiving imatinib for chronic myeloid leukemia. N Engl J Med 355:2408-2417

18. Kwak EL, Bang YJ, Camidge DR et al (2010) Anaplastic lymphoma kinase inhibition in non-small-cell lung cancer. N Engl J Med 363:1693-1703

19. Tang Y, Axelsson AS, Spegel P et al (2014) Genotype-based treatment of type 2 diabetes with an $\alpha 2 \mathrm{~A}$-adrenergic receptor antagonist. Sci Transl Med 6:257ra139

20. Boule NG, Weisnagel SJ, Lakka TA et al (2005) Effects of exercise training on glucose homeostasis: the HERITAGE family study. Diabetes Care 28:108-114

21. Bohm A, Weigert C, Staiger H, Haring HU (2016) Exercise and diabetes: relevance and causes for response variability. Endocrine $51: 390-401$

22. Bouchard C, Blair SN, Church TS et al (2012) Adverse metabolic response to regular exercise: is it a rare or common occurrence? PLoS One 7, e37887

23. Weyrich P, Stefan N, Haring HU, Laakso M, Fritsche A (2007) Effect of genotype on success of lifestyle intervention in subjects at risk for type 2 diabetes. J Mol Med 85:107-117

24. Perusse L, Rice T, Province MA et al (2000) Familial aggregation of amount and distribution of subcutaneous fat and their responses to exercise training in the HERITAGE family study. Obes Res 8:140-150

25. Bouchard C, Tremblay A, Despres JP et al (1994) The response to exercise with constant energy intake in identical twins. Obes Res 2: 400-410

26. Bouchard C, Tremblay A, Despres JP et al (1990) The response to longterm overfeeding in identical twins. N Engl J Med 322:1477-1482
27. Ahmad S, Rukh G, Varga TV et al (2013) Gene $\times$ physical activity interactions in obesity: combined analysis of 111,421 individuals of European ancestry. PLoS Genet 9, e1003607

28. Andreasen CH, Stender-Petersen KL, Mogensen MS et al (2008) Low physical activity accentuates the effect of the FTO rs9939609 polymorphism on body fat accumulation. Diabetes 57:95-101

29. Vimaleswaran KS, Li S, Zhao JH et al (2009) Physical activity attenuates the body mass index-increasing influence of genetic variation in the FTO gene. Am J Clin Nutr 90:425-428

30. Rampersaud E, Mitchell BD, Pollin TI et al (2008) Physical activity and the association of common FTO gene variants with body mass index and obesity. Arch Intern Med 168:1791-1797

31. Franks PW, Jablonski KA, Delahanty LM et al (2008) Assessing genetreatment interactions at the FTO and INSIG2 loci on obesity-related traits in the diabetes prevention program. Diabetologia 51:2214-2223

32. Kilpeläinen TO, Qi L, Brage S et al (2011) Physical activity attenuates the influence of $F T O$ variants on obesity risk: a meta-analysis of 218,166 adults and 19,268 children. PLoS Med 8, e1001116

33. Young AI, Wauthier F, Donnelly P (2016) Multiple novel gene-byenvironment interactions modify the effect of $F T O$ variants on body mass index. Nat Commun 7:12724

34. Gerken T, Girard CA, Tung YC et al (2007) The obesity-associated FTO gene encodes a 2-oxoglutarate-dependent nucleic acid demethylase. Science 318:1469-1472

35. Grunnet LG, Brons C, Jacobsen S et al (2009) Increased recovery rates of phosphocreatine and inorganic phosphate after isometric contraction in oxidative muscle fibers and elevated hepatic insulin resistance in homozygous carriers of the A-allele of FTO rs9939609. J Clin Endocrinol Metab 94:596-602

36. Smemo S, Tena JJ, Kim KH et al (2014) Obesity-associated variants within FTO form long-range functional connections with IRX3. Nature 507:371-375

37. Claussnitzer M, Dankel SN, Kim KH et al (2015) FTO obesity variant circuitry and adipocyte browning in humans. N Engl J Med 373:895-907

38. Xiang L, Wu H, Pan A et al (2016) FTO genotype and weight loss in diet and lifestyle interventions: a systematic review and metaanalysis. Am J Clin Nutr 103:1162-1170

39. Palla L, Higgins JP, Wareham NJ, Sharp SJ (2010) Challenges in the use of literature-based meta-analysis to examine geneenvironment interactions. Am J Epidemiol 171:1225-1232

40. Livingstone KM, Celis-Morales C, Papandonatos GD et al (2016) FTO genotype and weight loss: systematic review and metaanalysis of 9563 individual participant data from eight randomised controlled trials. BMJ 354:i4707

41. Papandonatos GD, Pan Q, Pajewski NM et al (2015) Genetic predisposition to weight loss and regain with lifestyle intervention: analyses from the diabetes prevention program and the look AHEAD randomized controlled trials. Diabetes 64:4312-4321

42. Nettleton JA, Follis JL, Ngwa JS et al (2015) Gene $\times$ dietary pattern interactions in obesity: analysis of up to 68317 adults of European ancestry. Hum Mol Genet 24:4728-4738

43. Goran MI, Poehlman ET (1992) Endurance training does not enhance total energy expenditure in healthy elderly persons. Am J Phys 263:E950-E957

44. Shephard RJ (2003) Limits to the measurement of habitual physical activity by questionnaires. Br J Sports Med 37:197-206

45. Lobelo F, Kelli HM, Tejedor SC et al (2016) The wild wild west: a framework to integrate mhealth software applications and wearables to support physical activity assessment, counseling and interventions for cardiovascular disease risk reduction. Prog Cardiovasc Dis 58:584-594

46. Jakicic JM, Davis KK, Rogers RJ et al (2016) Effect of wearable technology combined with a lifestyle intervention on long-term weight loss: the IDEA randomized clinical trial. JAMA 316:1161-1171

47. Zeevi D, Korem T, Zmora N et al (2015) Personalized nutrition by prediction of glycemic responses. Cell 163:1079-1094 